\title{
El aprendizaje-servicio, una herramienta para el desarrollo profesional de la responsabilidad social del periodista
}

\author{
Carmen MARTA LAZO \\ Universidad de Zaragoza \\ cmarta@unizar.es
}

Patricia GonZÁlez AldeA

Universidad Carlos III de Madrid

patricia.gonzalez.aldea@uc3m.es

Recibido: $14 / 07 / 2012$

Aceptado: 23/10/2012

\section{Resumen}

El método del Aprendizaje-Servicio es muy útil en el contexto del Espacio Europeo de Educación Superior pues desarrolla competencias curriculares y profesionales del estudiante a través de un servicio a la comunidad. El estudio que presentamos tiene como objetivo mostrar los resultados obtenidos en un proyecto de Aprendizaje-Servicio llevado a cabo en el Grado de Periodismo de la Universidad de Zaragoza. La actividad de servicio consistió en realizar un reportaje de televisión que mostrara las acciones sociales de entidades sin ánimo de lucro. El estudiante tomaría así conciencia de la trascendencia de la profesión periodística como servicio público. Mediante un cuestionario de autoevaluación del servicio prestado los alumnos valoraron la actividad. Este artículo se enmarca en el "Proyecto de Innovación Docente de Aprendizaje-Servicio" de la Universidad de Zaragoza (PIIDUZ 2011.1).

Palabras clave: Aprendizaje-Servicio, Periodismo, servicio público, responsabilidad social, reportaje.

\section{Service-Learning, a tool for professional development of the journalist's social acccountability}

\begin{abstract}
The Service-Learning method is very useful in the context of the European Higher Education Area since it enhances the curricular and professional skills of the student through a community service. The present study aims to show the results in a Service-Learning project undertaken in the Bcahelor's Degree in Journalism at the University of Zaragoza. The service activity was based on recording a television report which showed the social actions of nonprofit organizations. Therefore, students would be aware of the implications of the journalistic profession as a public service. Students rated the activity by filling in a self-assessment form about the provided service. This article is part of the 'Service-Learning Teaching Innovation Project' at the University of Zaragoza (PIIDUZ 2011.1).

Keywords: Service-Learning, Journalism, public service, social accountability, report.

\section{Referencia normalizada}

MARTA LAZO, Carmen y GONZÁLEZ ALDEA, Patricia (2012): "El aprendizaje-servicio, una herramienta para el desarrollo profesional de la responsabilidad social del periodista". Estudios sobre el mensaje periodístico. Vol. 18, núm. especial noviembre, págs.: 577-588. Madrid, Servicio de Publicaciones de la Universidad Complutense.
\end{abstract}

Sumario: 1. Introducción; 1.1. Estado de la cuestión y fuentes. 2. Hipótesis. 3. Metodología y desarro1lo; 3.1. Diagnóstico; 3.2 Planificación; 3.3. Ejecución; 3.4. Puesta en común y lecciones aprendidas; 3.5 Autoevaluación del alumno. 4. Análisis de resultados; 4.1. Valoración de competencias desarrolladas en el ApS; 4.2. Calificación de los resultados de aprendizaje alcanzados; 4.3. Aportaciones de la actividad periodística en términos de servicio público; 4.4. Grado de interés de la actividad como futuro periodista. 5. Conclusiones. 6 . Referencias bibliográficas. 


\section{Introducción}

Este artículo se enmarca en el "Proyecto de Innovación Docente de Aprendizaje-Servicio"1 de la Universidad de Zaragoza (PIIDUZ 2011.1) aplicado en el curso 2011-2012 a los estudiantes del Grado de Periodismo. Se trata de una iniciativa novedosa dentro del ámbito universitario de los estudios de Comunicación, puesto que la metodología educativa conocida como Aprendizaje-Servicio (ApS) ha estado vinculada en España en gran medida a otros estudios, como Educación, Pedagogía, Filología, o Enfermería, y a experiencias en Bachillerato en torno a la educación para la ciudadanía. (Martín, Muñoz y Puig, 2006; Sanchez Martín, 2006; Puig, Gijón, Martín y Rubio, 2011).

El ApS es una herramienta no sólo eficaz para optimizar la calidad del aprendizaje académico, sino también para formar personas y profesionales socialmente responsables, algo aún más relevante si cabe en el caso del Periodismo dada la finalidad de servicio público que tiene la profesión, como recoge entre otras la Ley General de la Comunicación Audiovisual (2010)².

La universidad actual, en el contexto del Espacio Europeo de Educación Superior (EEES), debe reforzar la formación de una ciudadanía crítica, solidaria y socialmente responsable que cuando finalice el aprendizaje académico ejerza tanto como ciudadano, como profesional educado para la democracia.

En el ApS se proporciona además del aprendizaje para el estudiante un servicio a la comunidad. El destinatario es por tanto doble, el beneficio es mutuo, como subraya Andrew Furco (2002), director del Service-Learning Research and Development Center de la Universidad de California-Berkeley. La naturaleza recíproca del servicio (D' Arlach, Sánchez y Feuer, 2009: 6), la prosocialidad (Cecchi, 2006: 2-4) son la base del ApS. La valoración por ambas partes del proyecto y el servicio realizado es sin duda el indicador más claro del potencial del ApS.

En este artículo analizamos los resultados obtenidos en un ApS integrado, -que aúna los contenidos y objetivos curriculares de tres asignaturas: Empresa de Comunicación, Géneros Informativos en Televisión, y Producción de Informativos en Televisión-, con una actividad de servicio consistente en elaborar un reportaje de televisión sobre las acciones sociales y actividades solidarias de una entidad sin ánimo de lucro elegida por los alumnos. El objetivo era que el estudiante tomara así conciencia de la finalidad de servicio público, la trascendencia y la repercusión social del trabajo periodístico.

El proyecto se ha basado en los tres pilares metodológicos del ApS del modelo de Howard (2001): aprendizaje significativo, porque en el servicio los alumnos ponen en práctica conocimientos curriculares específicos de su carrera; servicio de calidad, porque responde a necesidades reales de los receptores y tiene un impacto real y cuantificable; aprendizaje social y cívico, porque el estudiante adquiere formación en valores que le llevan a participar más activamente y de forma más responsable en la sociedad.

1 Véase para más información web del proyecto: http://www.unizar.es/aprendizaje_servicio.

${ }^{2} \mathrm{Al}$ "servicio público de comunicación audiovisual" se refiere la ley cuando señala que la misión de este servicio esencial es "difundir contenidos que fomenten los principios y valores constitucionales", así como atender a "aquellos ciudadanos y grupos sociales que no son destinatarios de la programación mayoritaria". BOE n 79 (2010): Ley 7/2010, de 31 de marzo, General de la comunicación audiovisual, pp. 30157-30209. 
En los resultados del proyecto se muestra el auto-diagnóstico de los estudiantes a través de la autoevaluación del servicio, -tanto en lo relativo al aprendizaje curricular y experiencial, como al desarrollo de una mayor conciencia profesional sobre el servicio público periodístico.

\subsection{Estado de la cuestión y fuentes}

El "Service-Learning" o Aprendizaje-Servicio es un concepto acuñado hace ya décadas en Estados Unidos, con la primera conferencia nacional sobre ApS celebrada en Estados Unidos en 1969. En los fundamentos de esta metodología se encuentran desde los trabajos de la pionera Jane Addams en 1904 (Daynes y Longo, 2004: 5), al aprendizaje experiencial del pedagogo norteamericano John Dewey, o las corrientes del constructivismo social.

A aquella conferencia le siguieron acciones pioneras en América Latina en las Universidades de México, Costa Rica, y Colombia (Cecchi, 2006: 11). Sin embargo, en otros lugares, en especial en España, hasta esta última década no se ha producido una verdadera aproximación y un desarrollo de estos proyectos con carácter más generalizado (Martínez-Odría, 2007; Puig y Palos, 2007; Martínez (ed.), 2008; Puig (coord.) 2009; Francisco y Moliner, 2010; Folgueiras, Luna y Puig, 2013).

El Centro Latinoamericano de Aprendizaje y Servicio Solidario (CLAYSS) fundado en 2002 en Buenos Aires (Argentina) lleva una década impulsando el desarrollo de proyectos educativos solidarios. OPCION Latinoamérica es una red de gestión social vinculada a la Universidad a través de proyectos de aprendizaje fundada hace más de una década en Colombia.

En 2001 en la Pontificia Universidad Católica de Chile se creó un grupo con el fin de acercar el servicio al aula desde una perspectiva multidisciplinar. El proyecto terminó tres años después con un manual (Ramírez y Pizarro, 2005) para docentes sobre el ApS.

The National Service-Learning Clearinghouse (NSLC) se presenta como la fuente americana más completa de Service-Learning. Y en el contexto europeo en la última década, Folgueiras, Luna y Puig (2013: 5) recogen las principales aportaciones sobre ApS en el ámbito universitario.

En España, en Cataluña funciona el "Centro Promotor de Aprendizaje de Servicio" y "Zerbikas" es el Centro Promotor del Aprendizaje y Servicio Solidario en Euskadi. Francisco y Moliner (2010) aplicaron el ApS a las asignaturas de Educación cívica y social, Discursos audiovisuales y Cultura de paz y didáctica general, con el objetivo de construir ciudadanía crítica. En la obra editada por Martínez (2010) se recogen cuatro experiencias de ApS, tres desarrolladas en la Universidad de Barcelona y otra en Vic.

Frente a los diferentes tipos de experiencias solidarias, pero no necesariamente articuladas en función de una disciplina o aprendizaje académico, lo que aporta la fórmula del (ApS) es precisamente un vínculo entre la acción de servicio, la formación que está adquiriendo a través de los contenidos curriculares, y la labor profesional que desarrollará el estudiante. "No es una actividad extracurricular ubicada en el tiempo libre de los estudiantes (como lo sería un voluntariado o una acción solidaria espontánea), sino que es una forma de desarrollar los contenidos curriculares" (Ramírez y Pizarro, 2005:17). 
Durante las diferentes fases del proceso del ApS, -desde la detección de una necesidad social, la planificación del proyecto, el contacto con la entidad objeto del servicio, hasta la ejecución, finalización y valoración del servicio-, es importante para los estudiantes la comunicación y la guía de los docentes.

La adquisición de competencias objeto de la asignatura, la conexión con la práctica, el desarrollo de habilidades sociales y cooperativas, así como una mayor conciencia social y responsabilidad cívica, son las principales transformaciones en el alumnado tras la práctica de un ApS (Basinger and Bartholomew, 2006).

\section{Hipótesis}

En el ámbito de los estudios de Comunicación en España, no hemos encontrado bibliografía relacionada con prácticas en las que se aplique la metodología del Aprendizaje-Servicio. Teniendo en cuenta esta carencia, mediante este análisis intentaremos profundizar en una práctica en esta área de conocimiento.

Con el citado propósito, las hipótesis de las que parte nuestra investigación son las siguientes:

- la realización de una actividad de Aprendizaje-Servicio dotará a los alumnos de una mayor concienciación de la trascendencia y repercusión social de la profesión periodística.

- la participación de todos los alumnos mediante el aprendizaje colaborativo ayudará a conocer y poner en común las diferentes actividades que se desarrollan a lo largo de las diferentes fases del proceso de producción del reportaje.

- la autoevaluación de la práctica llevada a cabo servirá para que el alumno se replantee el compromiso ciudadano de su profesión y reflexione acerca del desarrollo personal y formativo que ha conseguido con la actividad.

- En cuanto a los receptores del servicio, será una forma de dar a conocer los beneficios sociales derivados de las acciones que desarrollan

\section{Metodología y desarrollo}

El ApS se concretó en la elaboración de un reportaje televisivo de cuatro minutos, en 10 grupos de 6 alumnos, relativo a la responsabilidad social de una empresa, institución u ONG, ejemplificándolo en el seguimiento de un caso real.

La metodología que utilizamos para la evaluación de la práctica desde la dimensión del Aprendizaje-Servicio ha sido cuantitativa, mediante la respuesta a un cuestionario por parte de los participantes en esta experiencia. El muestreo tiene un carácter intencional (participantes del Grado de Periodismo en el proyecto) y la muestra del estudio estuvo formada por 52 estudiantes $^{3}$.

Hasta llegar a dicha autoevaluación por parte de los alumnos los pasos fueron los siguientes:

\subsection{Diagnóstico}

El primer paso consistió en conocer mediante una exploración y diagnóstico las actividades que desarrolla una determinada entidad desde la perspectiva del servicio a la ciudadanía.

${ }^{3}$ El número total de estudiantes que participaron en esta actividad fue de 60, pero sólo cumplimentaron 52 el cuestionario porque el día que se distribuyó y contestó faltaron 8 personas a clase. 
Cada uno de los seis miembros del equipo debería considerar y valorar la importancia de la repercusión social que el tema de su reportaje podría tener de cara a dar a conocer las acciones sociales y solidarias de una determinada entidad. Y si respondía a una necesidad real de los receptores del servicio.

\subsection{Planificación}

Durante las cuatro semanas en las que los alumnos fueron elaborando su reportaje, contaron con la guía de los profesores. Tuvieron un total de cinco tutorías obligatorias, una cada semana con el fin de orientar y evaluar el proceso de realización de esta pieza, además de algunas tutorías añadidas que se prestaban bajo demanda.

En una primera tutoría, los alumnos presentaron a los profesores de "Géneros informativos en Televisión" y de "Empresa de la Comunicación" tres posibles temas, con su consiguiente documentación, para elegir el que estimaran más interesante, atendiendo a los criterios de noticiabilidad periodística, bajo el prisma de compromiso social.

Tras esta sesión, los temas que se decidieron fueron:

- Actividades de instituciones y asociaciones de colectivos vulnerables:

- El Refugio, OMSIDA, (Asociación para la ayuda a personas afectadas por VIH/SIDA de Zaragoza )

- ASPACE (Asociación Aragonesa de Parálisis Cerebral)

- ASAPME (Asociación Aragonesa Pro Salud Mental).

- Acciones sociales de Fundaciones de Cajas de Ahorros y aseguradoras:

- Caja de Ahorros de la Inmaculada de Aragón (CAI)

- DKV Seguros Médicos

- Barclays.

- Actuaciones de Federaciones con finalidad social o solidaria:

- FAS (Federación Aragonesa de Solidaridad)

- Federación de Asociaciones de Barrios de Zaragoza

- Fundación Proniño.

\subsection{Ejecución}

En una segunda tutoría, la profesora de "Producción de informativos en televisión" orientó a los grupos acerca del "gancho informativo".

En una tercera tutoría, desde "Géneros informativos en TV" se planteó la posible estructura del reportaje. Se trataba de plantearse en qué medida la forma, el contenido y la finalidad del reportaje respondía al servicio público periodístico.

En la cuarta sesión tutorial, se valoró el plan de rodaje y el guión.

En la quinta, se analizó el proceso de realización y las imágenes grabadas para dar paso a la edición final.

\subsection{Puesta en común y lecciones aprendidas}

Para completar y enriquecer la práctica, una vez grabados y editados todos los reportajes, se citó a cada grupo en una sesión, donde estaban presentes todos los alumnos de la clase, para exponer el modo en que elaboraron su reportaje. 
Los tres profesores involucrados en el proyecto de ApS formaron un tribunal en el que se valoró la pieza final desde las diferentes competencias que se plantearon en el marco de cada asignatura.

\subsection{Autoevaluación del alumno}

La actividad de Aprendizaje-Servicio concluyó con una autoevaluación de cómo se había despertado la conciencia solidaria desde una actividad basada en la práctica periodística.

\section{Análisis de resultados}

Una vez finalizado el curso, se les pasó el cuestionario, para que valoraran las competencias y resultados de aprendizaje alcanzados con la práctica, basándonos en la evaluación de competencias propuesto por Folgueiras, Luna y Puig (2013: 8) Su modelo de estudio del grado de satisfacción de los estudiantes universitarios está basado en 4 dimensiones clave: conocimiento e intencionalidad atribuida, valoración de la utilidad atribuida, valoración del proceso y proyección social.

El cuestionario se estructuró en cuatro partes:

a) Valoración de las competencias de las diferentes asignaturas desarrolladas en la práctica

b) Calificación de los resultados de aprendizaje alcanzados

c) Estimación de las principales aportaciones de la actividad en términos de servicio público periodístico

d) Evaluación del grado de interés de la actividad como futuro periodista

\subsection{Valoración de competencias desarrolladas en el ApS}

El promedio del resultado de las distintas competencias adquiridas alcanzó un notable. Los alumnos valoraron, con una media de un 8,2 , el conjunto de las competencias específicas desarrolladas en la actividad. La competencia que puntuaron de manera más destacada (con 8,4 puntos) fue la "Capacidad de comprender el concepto, características, elementos y tipología de los reportajes televisivos". El resto de las competencias alcanzaron en todos los casos una nota media superior a un 8. En el gráfico 1 , se observan las elevadas valoraciones obtenidas.

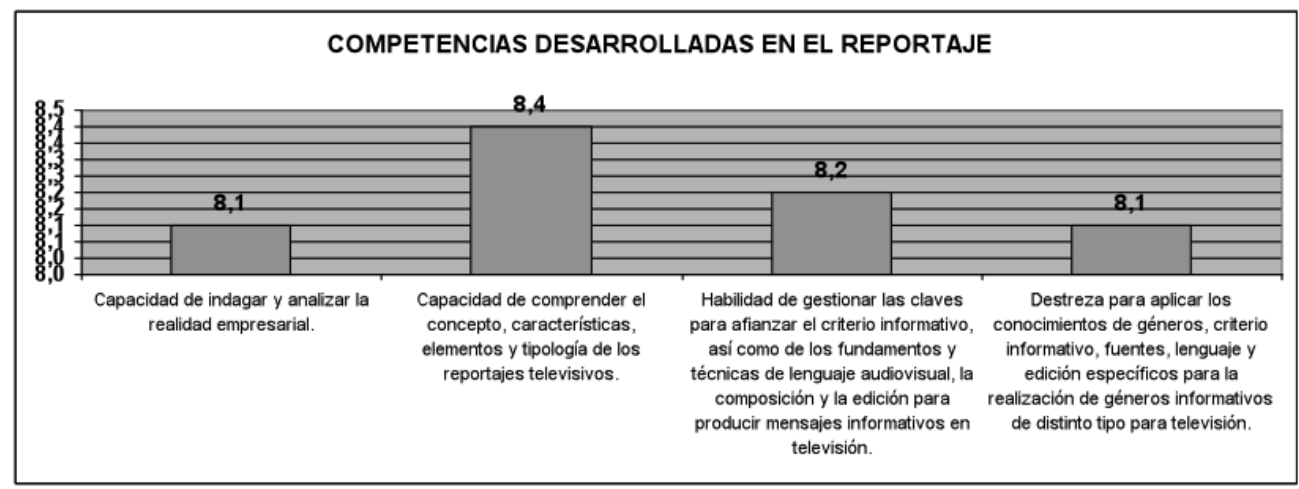

Gráfico 1. Valoración por los alumnos de las competencias adquiridas.

Fuente: elaboración propia 
A continuación, pasamos a representar de manera conjunta la distribución de frecuencias de cada competencia, con objeto de considerar todas las calificaciones contempladas en cada una de ellas.

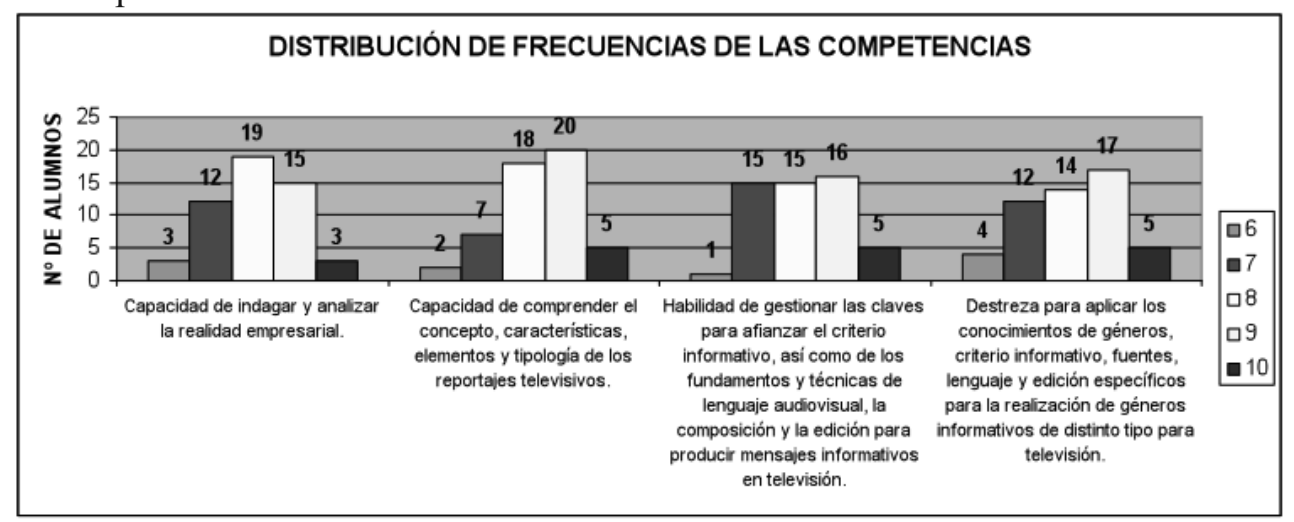

Gráfico 2. Puntuaciones otorgadas a cada competencia.

Fuente: elaboración propia

\subsection{Calificación de los resultados de aprendizaje alcanzados}

Por lo que respecta al segundo apartado, consistente en la valoración de los resultados de aprendizaje, el que presentó una mejor puntuación fue el de "Ser capaz de divulgar la actividad que desarrolla una institución desde el plano solidario" (con 8,8 puntos). Las otras competencias también fueron valoradas con un notable destacado, como se comprueba en el gráfico 3.

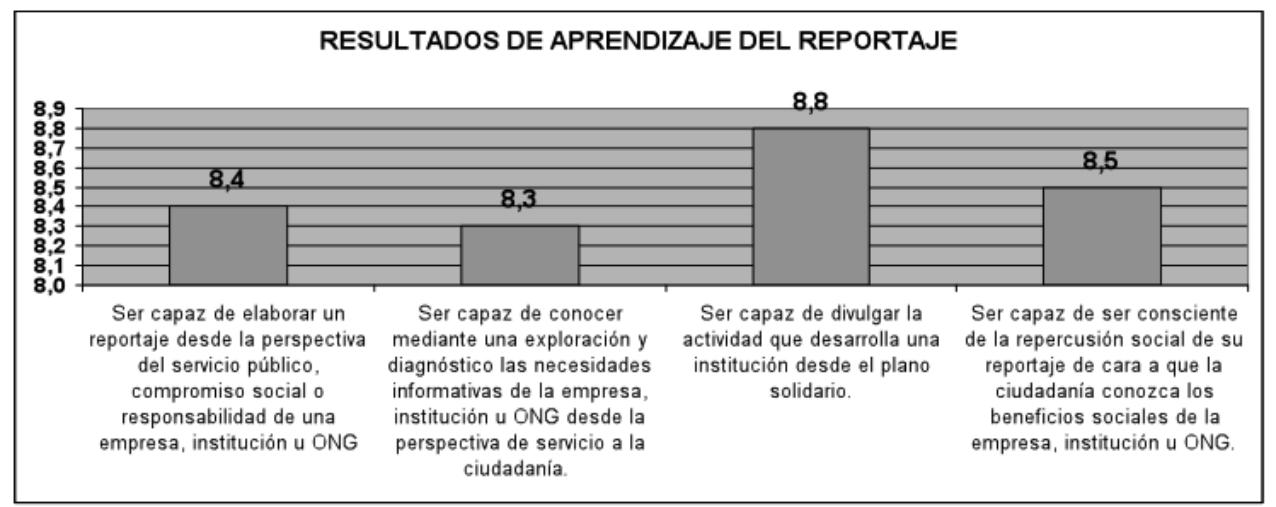

Gráfico 3. Valoración por los alumnos de los resultados de aprendizaje. Fuente: elaboración propia

En la distribución de frecuencias de los resultados de aprendizaje, observamos con más detalle las elevadas puntuaciones otorgadas. 


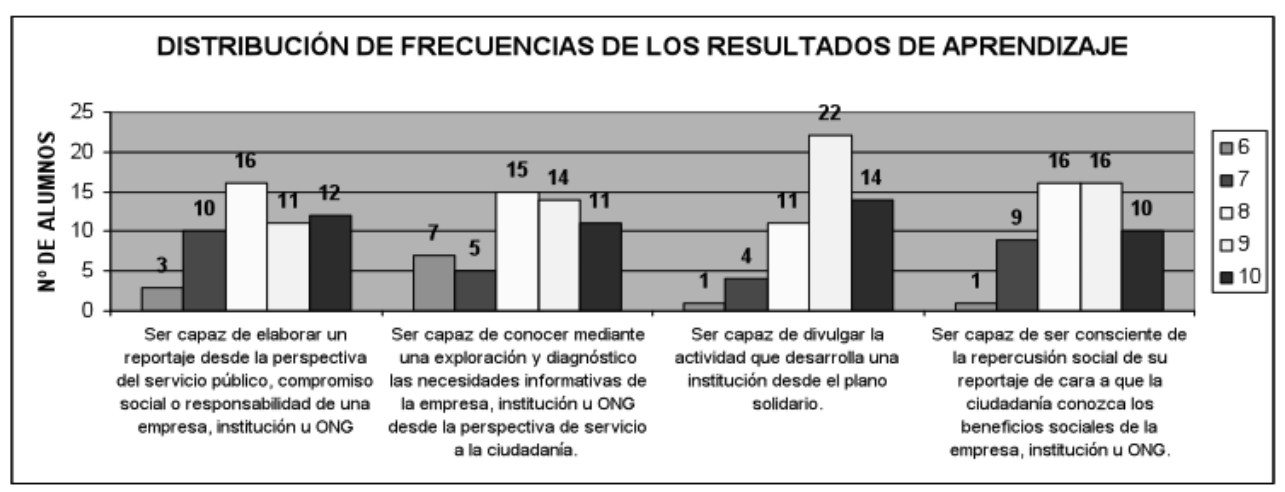

Gráfico 4. Puntuaciones otorgadas a cada resultado de aprendizaje.

Fuente: elaboración propia

\subsection{Aportaciones de la actividad periodística en términos de servicio público}

Propusimos al respecto una pregunta abierta ${ }^{4}$ para que los alumnos indicaran de forma libre aquellos aspectos que no se habían contemplaban en las preguntas anteriores. En los resultados de esta cuestión, hemos recopilado las respuestas más destacadas en términos de reiteración o énfasis que merezcan ser reseñables.

Las respuestas más repetidas pueden clasificarse en tres ejes principales:

a) Finalidad informativa, con tres perspectivas:

- Divulgar las acciones de la empresa/organización:

-"Dar conocer las actividades sociales de una empresa".

-"Dar protagonismo y voz a asociaciones para que se conozca su función”.

- Visibilizar a colectivos marginados en los medios:

-"Dar voz a un colectivo poco mediático".

-"Hacer visible a un colectivo no presente en los medios y conocer su verdadera función".

- Descubrir a la ciudadanía aspectos desconocidos de colectivos y empresas:

-"Concienciar a la gente de la existencia de este tipo de fundaciones".

-"Visión crítica. Qué sabe la sociedad y cuál es la realidad".

b) Finalidad ética, tomar conciencia de la responsabilidad social de la profesión periodística:

- "Saber transmitir una información con un enfoque social, resaltando la dimensión solidaria".

- "Tratar temas sensibles con profesionalidad".

c) Finalidad prospectiva, al haberse convertido en una actividad de "aprendizaje para la vida":

- "Fomentar valores positivos".

- "Una lección de vida, ahora miro con otros ojos a las ONG"

${ }^{4}$ La cuestión planteada era: "Señala las aportaciones que desde el punto de vista de Servicio Público periodístico has conseguido desarrollar con este reportaje". 
A continuación, recopilamos otras respuestas que nos parecen significativas. En algún caso, se plantea incluso el poder movilizador de la información social, en la más pura línea de la tradición norteamericana del "Service Journalism", -que más allá de una llamada a la solidaridad, busca cambiar actitudes, aproximarse a otros seres humanos, y/o ofrecer nuevas pautas vitales-, definido como "la información que aporta al receptor la posibilidad afectiva de acción y/o reacción. Aquella información, ofrecida oportunamente que pretende ser del interés personal del lector-oyenteespectador; que no se limita a informar sobre, sino para [...]" (Diezhandino, 1994: 89). Desde esta perspectiva, encontramos respuestas como las siguientes:

- "Mostrar una forma más de ayudar en la sociedad, puede servir de ejemplo y ayudar a los jóvenes".

- "Concienciar a la gente para que realice una actividad: movilizar".

Por último, se aludió al carácter profesionalizante de una práctica de estas características que promueve, según los estudiantes, un periodismo real.

\subsection{Grado de interés de la actividad como futuro periodista}

Para finalizar el cuestionario, incluimos un apartado en el que planteamos el grado de interés de esta actividad en relación con la profesión periodística para la que los alumnos se estaban preparando. La calificación media se situó en un 4,6 sobre 5, lo que también equivale a un sobresaliente destacado (9,2 sobre 10). Ningún alumno valoró el ejercicio como poco o nada interesante.

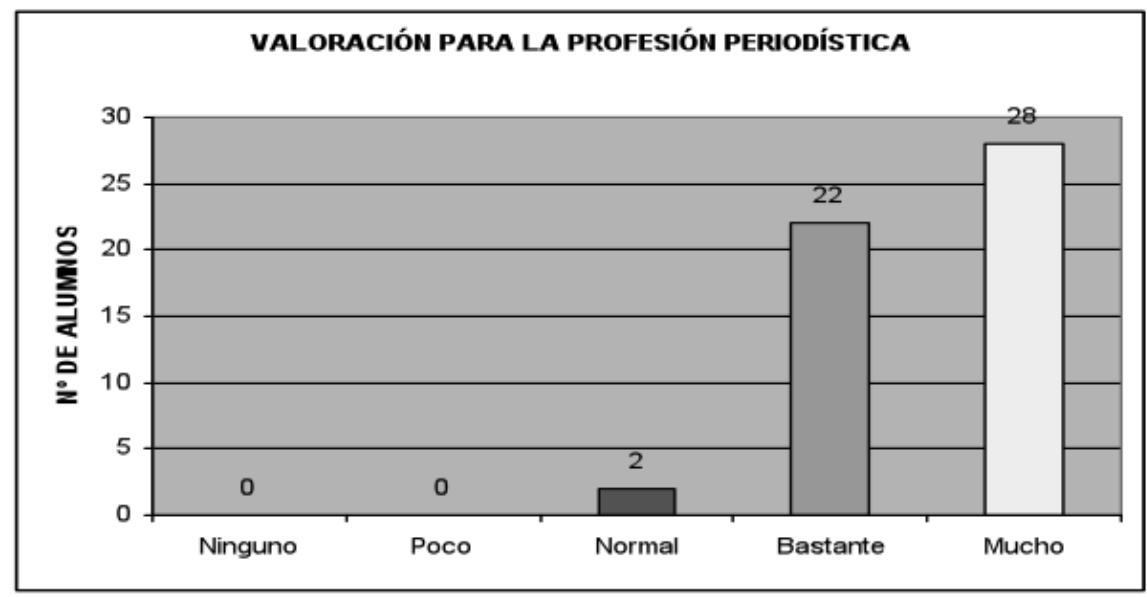

Gráfico 5. Grado de interés de la actividad para el futuro profesional. Fuente: elaboración propia

Una vez realizada la actividad se envió una copia del reportaje a las distintas organizaciones que habían servido como fuente y todos los colaboradores comentaron el interés del mismo para su organización, valorando positivamente el servicio.

Los resultados docentes alcanzados en la actividad, que se obtuvieron mediante las notas medias en las tres asignaturas, resultaron también satisfactorios. La mayo- 
ría de los alumnos obtuvieron un notable (un 53,8\%). El 34,6\% logró el sobresaliente, el 11,5\% aprobado, y cabe destacar que ningún grupo suspendió la actividad.

Por último, hay que añadir que todos los reportajes elaborados por los alumnos se colgarán en la web del proyecto de innovación con el fin de que los resultados trasciendan la propia aula.

\section{Conclusiones}

Las prácticas de Aprendizaje-Servicio (ApS) en las que se dan cita diferentes métodos de aprendizaje activo, como el aprendizaje experiencial, el cooperativo o el constructivismo, coinciden con los fundamentos pedagógicos demandados en el Espacio Europeo de Educación Superior (EEES).

El proyecto de Aprendizaje-Servicio realizado en el Grado de Periodismo de la Universidad de Zaragoza ha sido "significativo", porque ha puesto en práctica los conocimientos curriculares de las tres asignaturas de televisión; ha sido "un servicio de calidad", porque el reportaje ha respondido a necesidades reales de las entidades receptoras como ellas mismas han corroborado; y ha sido un "aprendizaje social y cívico", porque les ha servido tanto a nivel personal como profesional para mejorar en valores cívicos y tomar conciencia de la responsabilidad social de la profesión periodística, como han constatado los alumnos en las respuestas de su autoevaluación.

La producción audiovisual sirve a las entidades en las que se fundamenta el servicio como soporte de difusión de su actividad.

La realización de un reportaje con finalidad social sirve a los alumnos para el desarrollo de todas las competencias curriculares planteadas y para alcanzar los resultados de aprendizaje de manera exitosa, al tiempo que proporciona un servicio a la comunidad.

La integración en una misma práctica de competencias interdisciplinares permite englobar varios ejercicios en uno, lo que fomenta la calidad del ejercicio y el que los alumnos no se desgasten teniendo que elaborar numerosas prácticas, aspecto del que se quejan reiteradamente en el contexto del EEES.

El seguimiento del trabajo realizado a través de varias tutorías con los profesores ofrece a los alumnos orientación, tanto acerca de los aspectos técnicos y la vinculación de la teoría con la práctica, como en relación con la responsabilidad social del mismo.

El proceso de desarrollo de la actividad y su consiguiente autoevaluación ayuda a los alumnos a valorar y reflexionar sobre el compromiso social del periodismo. En general, los estudiantes estimaron como "muy interesante" la práctica atendiendo a su aplicación para el futuro profesional.

La publicación de los reportajes en la web del proyecto de innovación posibilita la difusión y permite que la práctica pueda retomarse por parte de investigadores, periodistas o universitarios para futuras elaboraciones audiovisuales.

\section{Referencias bibliográficas}

BASINGER, Nancy y BARTHOLOMEW, Keith (2006): "Service-Learning in Nonprofit Organizations: motivations, expectations, and outcomes", en Michigan Journal of Community Service Learning: http://quod.lib.umich.edu/m/mjcsl/3239521 $.0012 .202 / 1 /$-service-1earning-in-nonprofit-organizationsmotivations? view=image. 
CECCHI, Néstor Horacio (2006): “Aprendizaje Servicio en Educación Superior. La experiencia latinoamericana”, en Seminario Internacional Responsabilidad Social Universitaria: Aprendizaje Servicio, Universidad Central de Venezuela: http://www.documentacion.edex.es/docs/0406CECapr.pdf.

D'ARLACH, Lucía, SÁNCHEZ, Bernadette, y FEUER, Rachel (2009): "Voices from the community: a case for reciprocity in Service-Learning", en Michigan Journal of Community Service Learning: http://quod.lib.umich.edu/cgi/p/pod/dodidx/3239521.0016.101.pdf?c=mjcsl;idno=3239521.0016.101.

DAYNES, Gary y LONGO, Nicholas V. (2004): "Jane Addams and the Origins of Service-Learning Practice in the United States", en Michigan Journal of Community Service Learning: http://quod.lib.umich.edu/m/mjcsl/3239521.0011.101 ?rgn=main; view=fulltext.

DIEZHANDINO, María Pilar (1994): Periodismo de Servicio. Barcelona, Bosch.

FOLGUEIRAS, Pilar, LUNA, Esther y PUIG, Gemma (2013): “Aprendizaje y servicio: estudio del grado de satisfacción de estudiantes universitarios", en Revista de Educación: http://www.revistaeducacion.mec.es/doi/362_157.pdf.

FRANCISCO, Andrea y MOLINER, Lidón (2010): "El Aprendizaje Servicio en la Universidad: una estrategia en la formación de ciudadanía crítica", en Revista Electrónica Interuniversitaria de Formación del Profesorado: http://www.aufop.com /aufop/uploaded_files/articulos/1291992629.pdf.

FURCO, Andrew y BILLIG, Shelley H. (eds. (2002): Service-Learning: The Essence of the Pedagogie. Greenwich, Information Age Publishing.

HOWARD, Jeffrey (ed., 2001): Service-learning course design. Workbook. Michigan, Edward Ginsberg Center for Community Service, The University of Michigan.

MARTÍN, Jesús; MUÑOZ, Alex y PUIG; Josep María (2006): "Siete ejemplos para visualizar el aprendizaje servicio". Cuaderns de pedagogía, $\mathrm{n}^{\circ} 357$. Barcelona, Edita Wolters Kluwer España, pp.70-79.

MARTÍNEZ-ODRÍA, Arantzazu (2007): "Service-Learning o Aprendizaje-Servicio. La apertura de la escuela a la comunidad local como propuesta de educación para la ciudadanía", en Bordón: dialnet.unirioja.es/servlet/fichero_articulo?codigo $=2582784$.

MARTÍNEZ, Miquel (ed) (2010): Aprendizaje servicio y responsabilidad social de las Universidades. Barcelona, ICE y Ediciones Octaedro.

OHIO STATE UNIVERSITY (2004): "Creating Community Through Service-Learning. A faculty guide to creating service-learning courses at the Ohio State University", en Osu.edu: http://service-learning.osu.edu/_oldsite/history.php.

PUIG, Joseph María; GIJÓN, Mónica; MARTÍN, Xus y RUBIO, Laura (2011): "Aprendizaje-servicio y Educación para la Ciudadanía", en Revista de Educación: http://www.revistaeducacion.educacion.es/re2011/re2011_03.pdf.

PUIG, José María y PALOS, Josep (2006): “Rasgos pedagógicos del aprendizaje-servicio". Cuadernos de Pedagogía, n 357 . Barcelona, Praxis, pp. 60-63. 
PUIG, José María (coord.) (2009): Aprendizaje Servicio. Educación y compromiso cívico. Barcelona, Graó.

RAMÍREZ, Teresa y PIZARRO, Bernardita (2005): “Aprendizaje servicio Manual para docentes UC", en Pontificia Universidad Católica de Chile: http://www.documentacion.edex.es/docs/0403RAMapr.pdf

SANCHEZ MARTÍN, Jordi (2006): "El aprendizaje-servicio. Un instrumento de educación para la ciudadanía". Cuadernos de Pedagogía, no 357. Barcelona, Edita Wolters Kluwer España, pp. 56-59.

UNIVERSIDAD DE ZARAGOZA: Web del Proyecto de Innovación Aprendizaje, en: http://www.unizar.es/aprendizaje_servicio.

\section{Carmen MARTA LAZO}

Universidad de Zaragoza

cmarta@unizar.es

\section{Patricia GONZÁLEZ ALDEA}

Universidad Carlos III de Madrid

patricia.gonzalez.aldea@uc3m.es 